\title{
Retinoic acid rewires the adrenergic core regulatory circuitry of neuroblastoma but can be subverted by enhancer hijacking of MYC or MYCN
}

Mark W. Zimmerman ${ }^{1 \dagger}$, Adam D. Durbin ${ }^{1}$, Shuning He ${ }^{1}$, Felix Oppel ${ }^{1}$, Hui Shi ${ }^{1}$, Ting Tao ${ }^{1}$, Zhaodong $\mathrm{Li}^{1}$, Alla Berezovskaya ${ }^{1}$, Yu Liu ${ }^{2}$, Jinghui Zhang ${ }^{2}$, Richard A. Young ${ }^{3,4}$, Brian J. Abraham², A. Thomas $\operatorname{Look}^{1 \dagger}$

${ }^{1}$ Department of Pediatric Oncology, Dana-Farber Cancer Institute, Boston, MA, 02215

${ }^{2}$ Department of Computational Biology, St. Jude Children's Research Hospital, Memphis, TN 38105

${ }^{3}$ Whitehead Institute, Cambridge, MA, 02142

${ }^{4}$ Department of Biology, Massachusetts Institute of Technology, Cambridge, MA, 02142

†Email: markw_zimmerman@dfci.harvard.edu (M.W.Z.) and thomas_look@dfci.harvard.edu (A.T.L.)

Keywords: Neuroblastoma, transcription, retinoic acid, enhancer hijacking

Running title: Retinoic acid rewires the core regulatory circuitry of neuroblastoma 


\section{ABSTRACT}

Neuroblastoma cell identity depends on a core regulatory circuit (CRC) of transcription factors that collaborate with MYCN to drive the oncogenic gene expression program. For neuroblastomas dependent on the adrenergic $\mathrm{CRC}$, treatment with retinoids can inhibit cell growth and induce differentiation in both primary neuroblastomas and cell lines; however, the underlying mechanisms remain unclear. Here we show that when $M Y C N$-amplified neuroblastomas cells are treated with alltrans retinoic acid (ATRA), histone H3K27 acetylation and methylation become redistributed to decommission super-enhancers driving the expression of PHOX2B and GATA3, together with the activation of new super-enhancers that drive high levels of MEIS1, HIC1 and SOX4 expression. These findings indicate that treatment with ATRA can reprogram the enhancer landscape to collapse the adrenergic $\mathrm{CRC}$, resulting in downregulation of $M Y C N$ expression, while upregulating a new retinosympathetic CRC that causes proliferative arrest and sympathetic differentiation. Thus, we provide mechanisms that account for the beneficial effects of retinoids against high-risk neuroblastoma and explain the rapid downregulation of expression of $M Y C N$ despite massive levels of gene amplification. 


\section{INTRODUCTION}

Cell identity is established by transcriptional core regulatory circuits (CRCs) of cell-state specific transcription factors that are driven by super-enhancers and form an interconnected autoregulatory loop that coordinately regulates gene expression ${ }^{1,2}$. Throughout development, cell multipotency and differentiation are controlled by gene expression programs that are hierarchically regulated by the CRC and its extended regulatory network ${ }^{3-5}$. Lineage specification of the embryonic neural crest, in particular, is governed by a dynamic architecture of master transcription factors and regulatory networks that give rise to diverse cell lineages during development ${ }^{6}$.

Pediatric neuroblastoma, a neural-crest-derived tumor of the peripheral sympathetic nervous system ${ }^{7}$, arises most often in the adrenal medulla, where sympathetic progenitor cells can become transformed by aberrant expression of $M Y C N$ or $M Y C$ and fail to differentiate into mature sympathetic ganglia or neuroendocrine chromaffin cells ${ }^{8-11}$. Neuroblastomas in patients and experimental cell lines generally possess one of two CRC modules - the immature neural crest-like subtype, defined by high expression levels of the $P R R X 1, Y A P / T A Z$ and $A P-1$ transcription factor genes, or the more commonly observed adrenergic subtype, characterized by the expression of HAND2, ISL1, PHOX2B, GATA3, $T B X 2$, and $A S C L 1^{12-14}$. Current models suggest that the neural crest-derived progenitors give rise to the adrenergic cell state, culminating in terminally differentiated sympathetic neuronal cells and chromaffin neuroendocrine cells of the peripheral sympathetic nervous system ${ }^{15}$. In neuroblastomas with MYCN amplification, this oncogene stabilizes the adrenergic CRC to drive the expression of this transcriptional regulatory network and enforce a neuroblast cell state, while suppressing developmental signals that would normally induce differentiation or senescence ${ }^{16}$. However, MYCN overexpression also results in a vulnerability called transcriptional addiction and creates tumor-selective gene dependencies, which include the adrenergic CRC transcription factors that sustain high levels of MYCN gene expression ${ }^{17,18}$.

Retinoic acid-based therapeutics provide a clinical benefit for patients with neuroblastoma and other malignancies through their ability to suppress tumor growth and promote cell differentiation ${ }^{19-23}$. 
Adrenergic neuroblastoma cell lines treated with retinoids frequently exhibit phenotypes associated with neuronal differentiation ${ }^{24,25}$. Hence, we hypothesized that pharmacologically induced differentiation of neuroblastoma cells with retinoids depends on reprogramming of the adrenergic $\mathrm{CRC}$, leading to rapid downregulation of $M Y C N$ expression, even in the context of massive $M Y C N$ gene amplification. Using a combination of transcriptional and chromatin-based approaches, we examined the regulation of adrenergic $\mathrm{CRC}$ transcription factors in response to retinoic acid treatment and show that this regulatory loop is reprogrammed into a "retino-sympathetic" CRC, resulting in rapid downregulation of MYCN expression coupled with the induction of cell differentiation and proliferative arrest.

\section{RESULTS}

\section{Retinoic acid treatment inhibits tumor growth in MYCN transgenic zebrafish}

High-risk neuroblastoma patients receive isotretinoin (13-cis retinoic acid) as a maintenance therapy following high-dose chemotherapy with autologous stem cell transplantation, although the mechanistic basis for the efficacy of retinoid treatment is not well understood ${ }^{7}$. Using a faithful zebrafish model of $M Y C N$-driven neuroblastoma $(d b h: M Y C N)^{9}$, we tested the ability of isotretinoin to inhibit neuroblastoma progression in vivo. Three-week-old transgenic zebrafish exhibiting green fluorescent cell masses in the interrenal gland (analogous to the human adrenal medulla), were treated with DMSO or $2 \mu \mathrm{M}$ isotretinoin (Fig. 1a). Relative to the DMSO control group, zebrafish receiving isotretinoin had a median $70 \%$ reduction in tumor size after 6 days of treatment (Fig. 1b,c). 
a

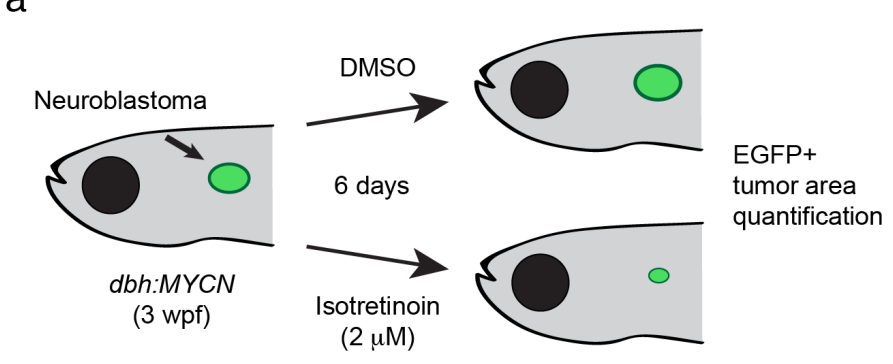

$\mathrm{b}$

C
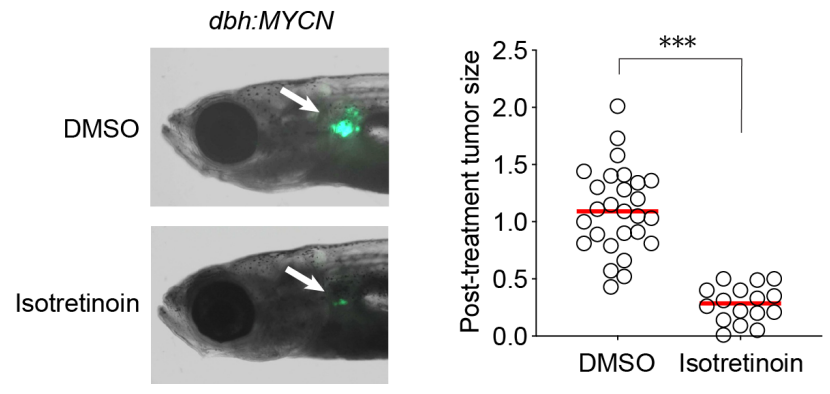

Figure 1. Retinoic acid suppresses the growth of transformed neuroblasts in vivo. a) Experimental design of the zebrafish tumor treatment assay. Tumor bearing dbh:MYCN transgenic zebrafish, 3 weeks post-fertilization (wpf), were treated with DMSO or Isotretinoin $(2 \mu \mathrm{M})$ for 6 days. Tumor size was quantified by the EGFP+ surface area. b) Representative images of $d b h: M Y C N$ transgenic zebrafish with EGFP+ sympathoadrenal cell hyperplasia (white arrows) that were treated with DMSO or $2 \mu \mathrm{M}$ isotretinoin for 6 days. b) Dot plot showing the distribution of post-treatment tumor size of DMSO $(n=26)$ or isotretinoin $(n=16)$ treatment in zebrafish, as quantified by EGFP cross sectional area. Red bar indicates the median value. ${ }^{* *} p<0.001$ by T-test.

\section{ATRA treatment of MYCN-amplified neuroblastoma cells collapses the adrenergic CRC}

To test the effects of retinoids on human $M Y C N$-amplified neuroblastoma cells, we treated two MYCN gene amplified neuroblastoma cell lines (BE2C and NGP) with all-trans retinoic acid (ATRA), an active metabolite of isotretinoin, for 6 days and then examined its effects on cell growth and viability. BE2C and NGP cell growth was significantly suppressed by 6 days of ATRA treatment relative to DMSOtreated control cells (Fig. 2a). Treatment with $5 \mu \mathrm{M}$ ATRA induced strong phenotypic changes in these cells, which included neurite outgrowth and upregulation of the structural proteins encoded by fibronectin (FN1), $\beta 3$-tubulin (TUBB3) and vimentin (VIM) (Fig. 2b). These findings are consistent with 
previous studies reporting the ability of retinoids to induce differentiation in MYCN-transformed neuroblastoma cells ${ }^{26,27}$.

Cell state and fate specification depend on precisely controlled gene expression programs, which are often under the control of an autoregulatory loop involving key transcription factors, called the core regulatory circuitry or $\mathrm{CRC}^{2}$. Thus, we investigated whether ATRA treatment affected the expression levels of members of the $M Y C N$-driven adrenergic CRC and its extended regulatory network, which program the malignant cell state in most $M Y C N$-amplified neuroblastoma cells ${ }^{18}$. Western blotting demonstrated that the CRC transcription factors MYCN, GATA3, PHOX2B were progressively downregulated in ATRA-treated cells, while others, such as HAND2 and ISL1, remained stable for up to 6 days after continuous treatment (Fig. 2c). This was unexpected because within a feed-forward autoregulatory loop, each transcription factor depends upon each of the others for its high levels of expression. We will demonstrate in the next section that this apparent paradox is explicable because HAND2 and ISL1 are retained as part of the new CRC that forms as the adrenergic CRC collapses in ATRA-treated cells.

a

$\mathrm{BE} 2 \mathrm{C}$

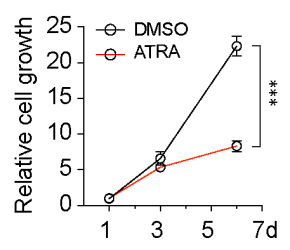

NGP

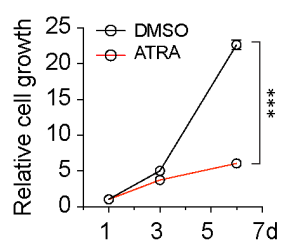

b

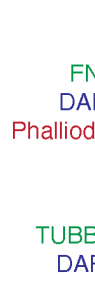

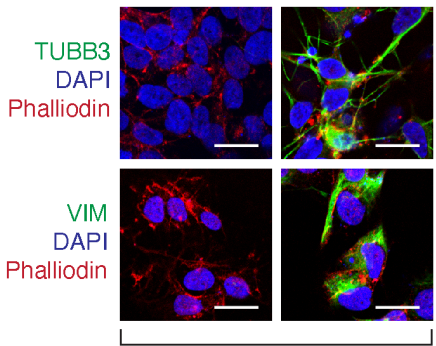

(MYCN-amplified)

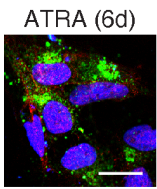

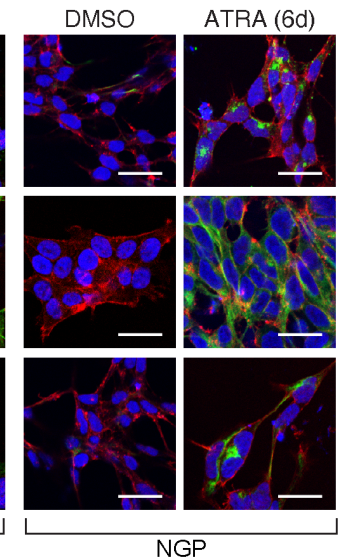

(MYCN-amplified)

C

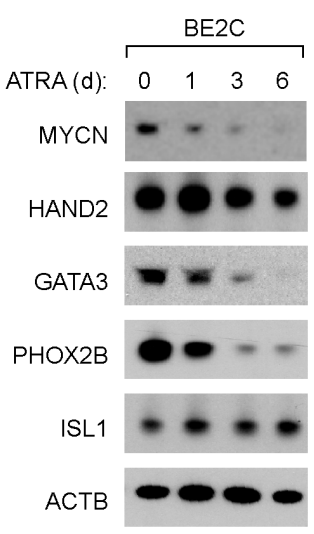

Figure 2. ATRA suppresses neuroblastoma cell growth and decreases protein levels of MYCN, GATA3 and

PHOX2B. a) Cell growth time course for BE2C and NGP cells comparing treatment with DMSO or $5 \mu \mathrm{M}$ all-trans retinoic 
bioRxiv preprint doi: https://doi.org/10.1101/2020.07.23.218834; this version posted July 24, 2020. The copyright holder for this preprint (which

was not certified by peer review) is the author/funder, who has granted bioRxiv a license to display the preprint in perpetuity. It is made available under aCC-BY-NC-ND 4.0 International license.

acid (ATRA) for 6 days; ${ }^{* *} \mathrm{p}<0.001$ by ANOVA and T-test at 6 days. b) Confocal images of BE2C and NGP neuroblastoma cells treated with DMSO or $5 \mu \mathrm{M}$ ATRA for 6 days. Cells were stained with fibronectin (FN1), $\beta 3$-tubulin (TUBB3) or vimentin (VIM) (green) and counterstained with DAPI (blue) and phalloidin (red). Scale bar indicates $25 \mu \mathrm{m}$. c) Western blot assay for CRC transcription factor levels (MYCN, HAND2, GATA3, PHOX2B, and ISL1) in BE2C cells treated with $5 \mu \mathrm{M}$ ATRA for $0,1,3$ and 6 days. ACTB is used as a protein loading control.

Using an ERCC spike-in normalized RNA-seq approach comparing cells after 6 days of DMSO or ATRA treatment, we observed dramatic changes in gene expression in ATRA-treated BE2C and NGP cells, with substantial downregulation of a subset of transcripts, including MYCN, GATA3 and PHOX2B, as shown in the violin plot in Fig. 3a. Notably, the expression of MYCN in ATRA-treated BE2C cells was reduced by more than $50 \%$, while the MYCN transcript became undetectable in NGP cells (Fig. 3b). Focusing on the highly expressed members of the adrenergic gene set described in recent studies ${ }^{12,13}$, we noted downregulated expression of many adrenergic CRC-regulated transcription factors, including the subset highlighted in red, which are experimentally validated members of the adrenergic CRC and tumor-selective gene dependencies (Fig. 3c) ${ }^{18}$. Note that some of the adrenergic transcription factors maintained high levels of expression despite a collapse of the adrenergic CRC (shown at the top of the bar graph in Fig 3c). As indicated by our Western blot results (Fig 2c), these exceptions reflect the inclusion of a subset of the genes in the new CRC that forms in response to ATRA treatment, as will be pursued in more detail in the next section.

Since CRC transcription factors facilitate the activity of gene enhancers, we examined whether these changes were associated with altered enhancer activity throughout the genome. We performed ChIP-seq for H3K27ac and H3K27me3 in BE2C and NGP cells treated with DMSO or ATRA for 12 days and examined changes in the cis-regulatory regions associated with differentially expressed genes. CRC transcription factors are associated with long stretches of H3K27ac-enriched enhancer peaks, termed super-enhancers, that are capable of driving high levels of gene expression at their target promoters ${ }^{2,28}$. The GATA3 and $P H O X 2 B$ genes, which both showed reduced transcript levels 
after treatment with ATRA, lost H3K27ac enrichment in their associated enhancer regions and gained H3K27me3 modifications at their promoters, which are histone modification patterns associated with chromatin silencing (Fig. 3c,d) ${ }^{29}$. Thus, ATRA-mediated differentiation of neuroblasts coincides with decreased expression of key CRC adrenergic transcription factors with corresponding changes in histone marks leading to repression of transcription.
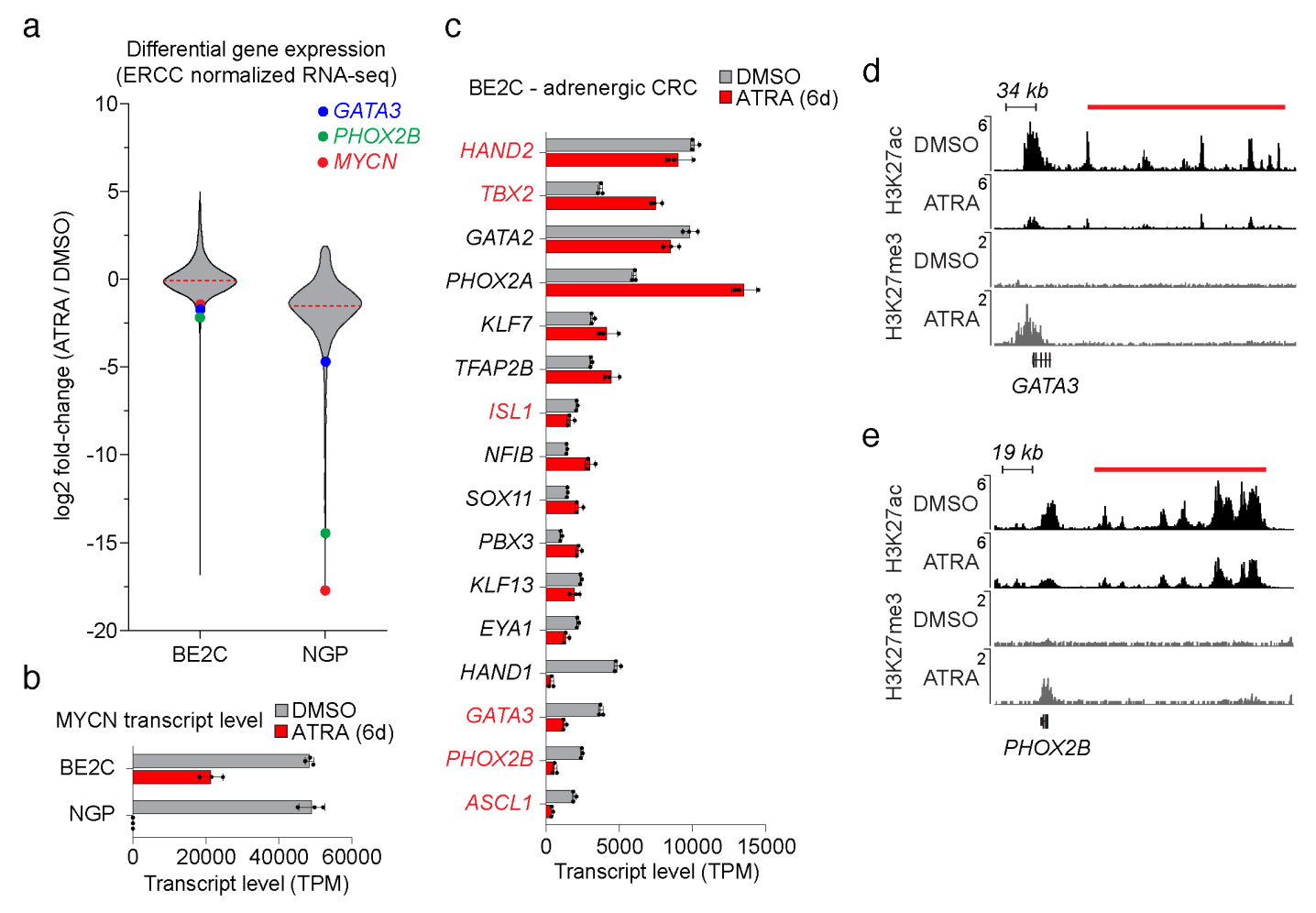

Figure 3. Effect of ATRA treatment on the adrenergic CRC of $M Y C N$-amplified neuroblastoma. a) Violin plot illustrating log2-fold changes (ATRA/DMSO) among all expressed genes (base mean TPM > 10) when assayed by spike-in normalized RNA-seq in BE2C and NGP (MYCN-amplified) cells. Changes are highlighted for MYCN (red), GATA3 (blue) and $P H O X 2 B$ (green), all of which were reduced at the protein level as well. b) Expression level of MYCN in BE2C and NGP cells treated with DMSO (grey) or $5 \mu \mathrm{M}$ ATRA (red) for 6 days. c) Expression levels of adrenergic CRC transcription factor genes in BE2C cells treated with DMSO (grey) or $5 \mu$ M ATRA (red) for 6 days. Neuroblastoma tumor-specific transcriptional dependencies are highlighted in red. d,e) Normalized ChIP-seq tracks for H3K27ac and H3K27me3 depicting super-enhancers associated with the GATA3 (d) and PHOX2B (e) gene loci in BE2C cells. Cells were treated with $5 \mu \mathrm{M}$ 
ATRA for 12 days; horizontal red bars indicate super-enhancers called by H3K27ac in DMSO-treated BE2C cells. ChIP-seq read densities (y axis) were normalized to reads per million reads sequenced from each sample.

\section{ATRA induces formation of a new CRC resulting in differentiation of neuroblastoma cells}

To test the hypothesis that ATRA treatment induces a new CRC that replaces the adrenergic CRC, we examined the genes encoding transcription factors that are upregulated due to prominent superenhancers that form after the start of ATRA treatment. In many cases, these super-enhancers were formed in gene regulatory regions that were not present before ATRA treatment. Several of the transcription factor genes that are most upregulated after treatment with ATRA - SOX4, MEIS1, and HIC1 - were associated with super-enhancers following ATRA treatment in both BE2C and NGP cells (Fig. 4a). Thus, the levels of H3K27ac modification in regulatory regions were enriched reflecting the formation of super-enhancers that drive expression of these genes in ATRA-treated BE2C and NGP cells (Fig. 4b). This indicates the emergence of a new CRC induced by ATRA treatment, in a process that is initiated by the ATRA ligand as an agonist of the RARA protein, which allows the transcription factor to form complexes with RXR and bind to its specific sites in DNA. Since antibodies are available for RARA and MEIS1 transcription factors that work well for immunoprecipitating bound chromatin, we performed ChIP-seq for these targets in BE2C cells treated with ATRA for 12 days. In addition to genome-wide binding to enhancers, as well as super-enhancers that regulate their own expression, RARA and MEIS1 occupied the super-enhancers regulating HAND2, ISL1 and TBX2, accounting for the continued high levels of expression of these genes, which were already expressed as members or targets of the adrenergic CRC network (Fig. 4c). We have termed this retinoid-induced CRC and its corresponding cell state in neuroblastoma the "retino-sympathetic" $\mathrm{CRC}$, to distinguish it from the adrenergic and neural-crest-like CRCs that were previously identified ${ }^{12,13}$.

Treatment with ATRA has been reported to downregulate the expression of MYCN and induce cell cycle arrest with either differentiation or apoptosis in $M Y C N$-amplified neuroblastoma cells ${ }^{26}$. Our results indicate that the collective activity of the adrenergic CRC, including HAND2, ISL1, PHOX2B, 
GATA3, ASCL1, and TBX2, is essential for maintaining the high oncogenic expression level of $M Y C N$, likely through activation of enhancers associated with the MYCN gene that are included in the amplified sequences $^{30}$. Loss of GATA3, PHOX2B and ASCL1 expression causes the adrenergic CRC to collapse after ATRA treatment, as noted in previous sections, accompanied by formation of the new retinosympathetic $\mathrm{CRC}$. Our results further indicate that the retino-sympathetic $\mathrm{CRC}$, which includes the transcription factors $R A R A, H I C 1$, SOX4 and MEIS1, is not capable of activating native MYCN enhancers included within its amplicon, so MYCN levels are rapidly down-regulated despite the high levels of amplification of the gene (Fig. 4d). MYCN is known to be a strong dependency factor in neuroblastoma with amplified $M Y C N^{18,31}$, accounting for the fact that neuroblastoma cells either undergo apoptosis or stop proliferating and undergo changes in gene expression consistent with terminal differentiation whenever the adrenergic CRC is dismantled and MYCN levels fall.

a

BE2C - retino-sympathetic CRC $\square D M S O$
$\square \operatorname{ATRA}(6 \mathrm{~d})$

HAND2 ...:

$T B \times 2 \longrightarrow$.

SOX $4 \longrightarrow$

TBX3 $\square$

$0 \quad 5000 \quad 1000015000$

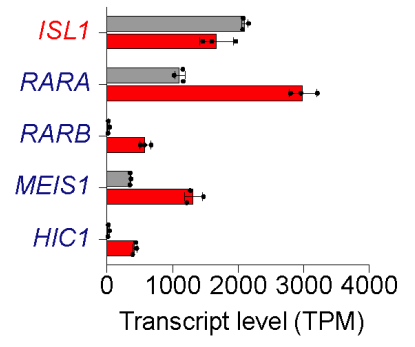

b

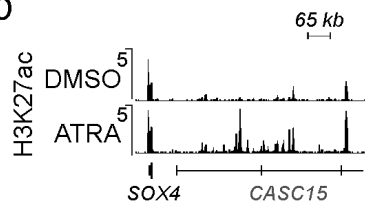

C

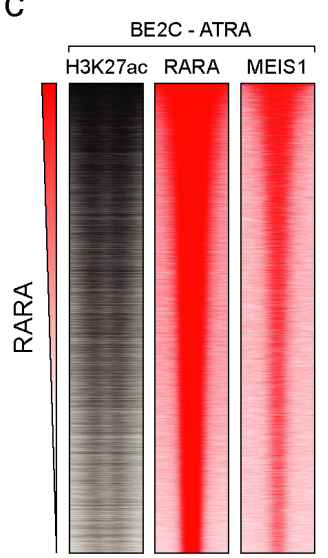

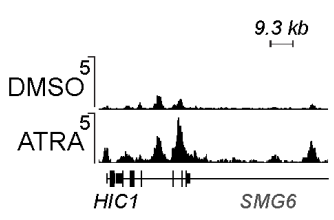

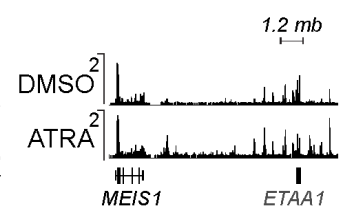

d

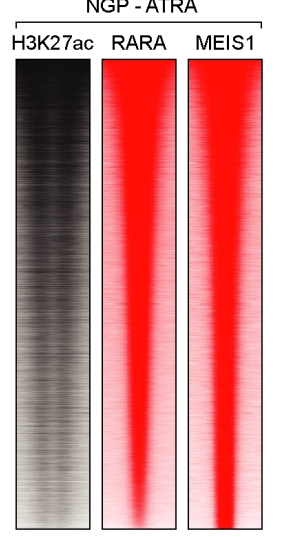

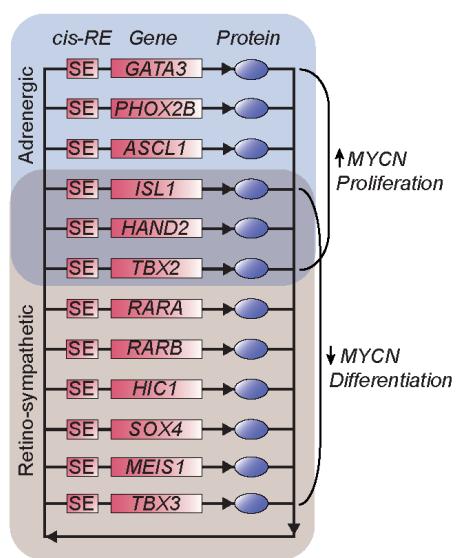

Figure 4. Cellular reprogramming by ATRA results in transition to a retino-sympathetic CRC transcriptional network. a) Transcript levels (TPM) of transcription factor genes upregulated in BE2C cells by treatment with ATRA (5 $\mu$ M) for 6 days. Transcriptional dependencies carried over from the adrenergic CRC are highlighted in red, and upregulated transcription factors associated with super-enhancers in ATRA-treated BE2C and NGP cells are highlighted in blue. b) 
Normalized ChIP-seq tracks depicting enrichment of H3K27ac modification in the regions surrounding the SOX4, HIC1 and MEIS1 gene loci in BE2C and NGP cells with DMSO or ATRA for 12 days. ChIP-seq read densities (y axis) were normalized to reads per million reads sequenced. c) Genome-wide co-occupancy for H3K27ac, RARA and MEIS1 in ATRAtreated BE2C and NGP cells as determined by ChIP-seq. Genomic regions (rows) were defined as those enriched in sequencing reads for at least one target and are ranked by the RARA signal therein. d) CRC transcription factors form an interconnected coregulatory loop, and treatment of adrenergic neuroblastoma cells with ATRA suppressed the expression and activity of GATA3, PHOX2B and ASCL1 while leading to the acquisition of RARA, RARB, SOX4, MEIS1, HIC1 and TBX3 as new members of the retino-sympathetic CRC. Regulatory elements and gene loci are denoted by rectangles, and proteins by oval symbols.

\section{ATRA resistance due to enhancer hijacking by the MYCN or MYC oncogenes}

A subset of neuroblastoma cell lines that express high levels of $M Y C$ or $M Y C N$ do not respond to treatment with retinoids ${ }^{32-34}$. One example is NBL-S, which expresses high levels of MYCN but does not have a high $M Y C N$ copy number. Instead, this cell line harbors a $t(2: 4)$ chromosomal translocation, which repositions the HAND2 super-enhancer in close genomic proximity to MYCN on one allele - a phenomenon known as enhancer hijacking ${ }^{11}$. Expression of HAND2 was not abolished in NBL-S by treatment with ATRA, and its associated super-enhancer remained stable when assayed by ChIP-seq (Fig. 5a). This outcome is consistent with the results in MYCN-amplified BE2C and NGP cells, where ATRA treatment had minimal effects on the stability of enhancers associated with HAND2.

In BE2C and NGP cells, the MYCN protein level was almost completely depleted by 6 days of treatment with ATRA; however, NBL-S cells treated with ATRA showed sustained levels of MYCN protein at both 3 and 6 days (Fig. 5b). Thus, because the retino-sympathetic CRC binds and activates the HAND2 super-enhancer, MYCN is still expressed at high levels in ATRA-treated NBL-S cells, which are blocked from differentiating and continue to proliferate as neuroblasts despite ATRA-induced activation of DNA binding by the RARA transcription factor. Several members of the retino-sympathetic CRC showed elevated transcript levels after ATRA treatment in these cells, but differentiation does not proceed in the context of sustained high levels of MYCN expression driven by the HAND2 enhancer. 
Similar results were observed with the high MYC-expressing cell line SKNAS, which harbors a $t(4: 8)$ rearrangement that repositions the HAND2 super-enhancer to drive high levels of expression of the MYC gene (Fig. 5b). Cell cycle phase distributions were analyzed before and after treatment with ATRA by flow cytometry measurements of cellular DNA content after propidium iodide staining. In contrast to BE2C and NGP cells, which became blocked in G1 phase, NBL-S and SKNAS cells treated with ATRA continued to enter $\mathrm{S}$ phase and progress through the cell cycle, consistent with the continued cell proliferation by these MYCN- or MYC-hijacked neuroblastoma cell lines (Fig. 5c,d).

a

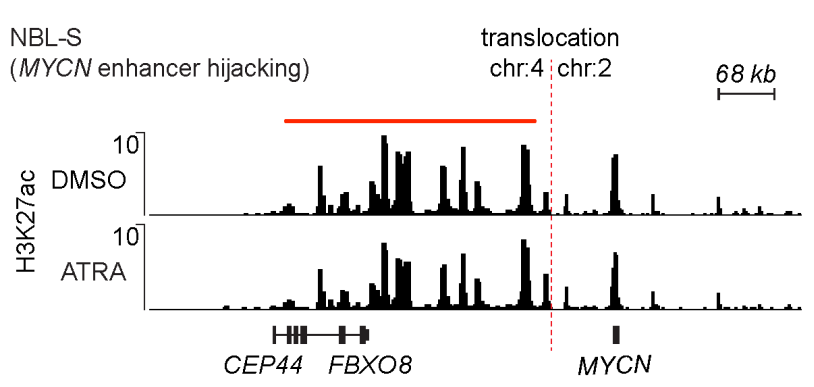

C

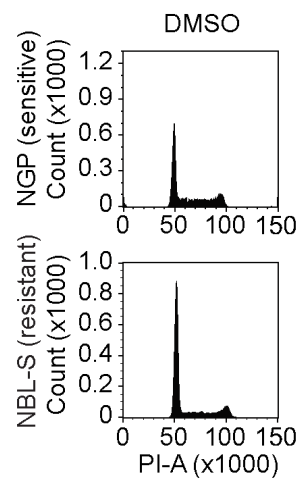

d
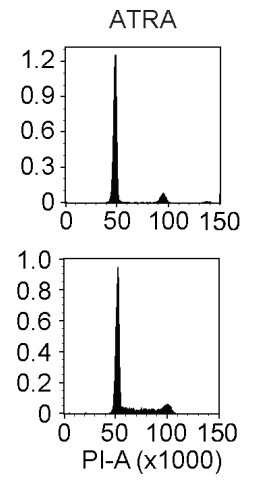

b

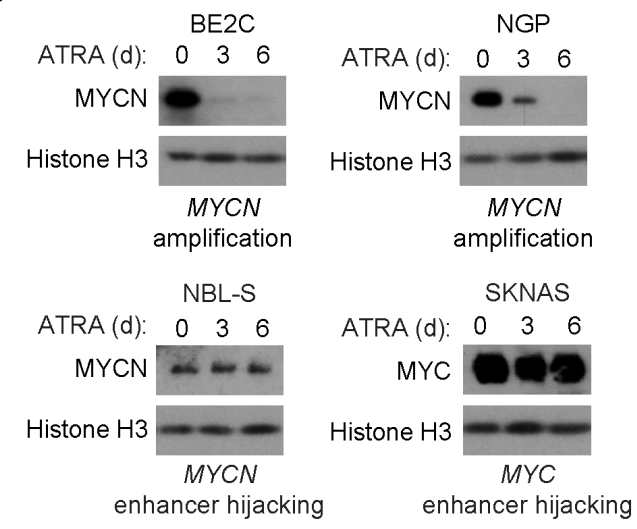

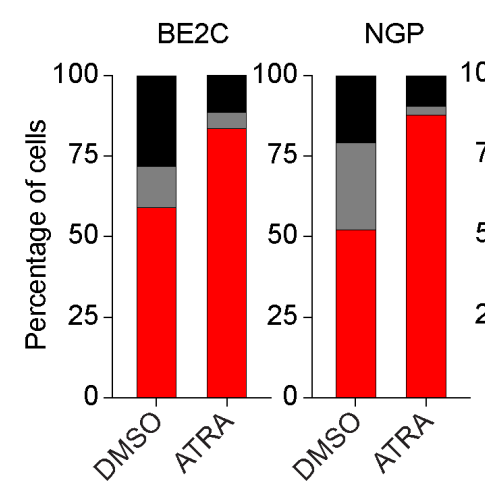

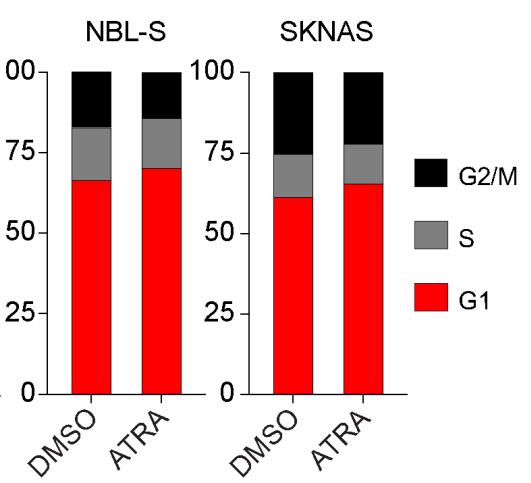

Figure 5. Hijacking the HAND2 super-enhancer subverts the suppression of $M Y C N$ or MYC expression following treatment with ATRA. a) H3K27ac ChIP-seq in NBL-S cells treated with DMSO or ATRA ( $5 \mu M)$ for 12 days, showing the genomic region surrounding the $t(2 ; 4)$ structural variation involving the $M Y C N$ gene locus. The region downstream of FBXO8 is a densely clustered super-enhancer that is translocated to within close proximity of the MYCN gene. b) Western blot assay measuring MYCN or MYC protein levels in BE2C, NGP, NBL-S and SKNAS cells following treatment with ATRA $(5 \mu \mathrm{M})$ for 0,3 and 6 days. c) FACS profiles depicting DNA content of NGP and NBL-S cells treated with DMSO or ATRA (5 
$\mu \mathrm{M}$ ) for 6 days. NGP cells (MYCN-amplified) became arrested in G1 phase, while NBL-S cells (MYCN enhancer hijacking) continued to progress from $\mathrm{G} 1$ to S phase. D) Bar graphs showing cell cycle distribution determined from hypotonic citrate propidium iodide (PI) staining of BE2C, NGP and NBL-S cells treated with DMSO or ATRA (5 $\mu$ M) for 6 days.

\section{DISCUSSION}

13-cis retinoic acid is a clinically important component of the current treatment approach for pediatric neuroblastoma and has been shown to inhibit cell growth and induce differentiation when tested in vitro using many different neuroblastoma cell lines ${ }^{24,27,35-37}$. We sought to explain why ATRA, the active metabolite of 13-cis retinoic acid, exerts these effects in neuroblastoma. Here we show that ATRA is capable of reprogramming the cell state of neuroblastoma, by fundamentally altering the core regulatory transcription factors that initiate and maintain the adrenergic gene expression program required for the tumorigenicity of these cells ${ }^{12,13,18}$.

The adrenergic CRC consists of an interdependent autoregulatory expression loop that includes HAND2, ISL1, PHOX2B, GATA3, ASCL1 and TBX2, and is essential to drive the expression of MYCN and facilitate the growth and survival of $M Y C N$-amplified neuroblastoma cells ${ }^{14,18}$. Treating $M Y C N-$ amplified neuroblastoma cells with ATRA resulted in rapid downregulation of MYCN expression, loss of H3K27ac chromatin modifications associated with active super-enhancers within the PHOX2B and GATA3 gene loci, and the acquisition of H3K27me3 chromatin silencing modifications of the promoters of these genes, which together lead to decreased expression levels of members of the adrenergic CRC. Despite the massively increased copy number of $M Y C N$ in these cells, $M Y C N$ expression levels were extremely sensitive to ATRA-treatment and became profoundly downregulated following collapse of the $\mathrm{CRC}$ required to drive its expression. Thus, one effect of ATRA treatment is collapse of the adrenergic CRC due to direct or indirect repression of the MYCN, PHOX2B and GATA3 genes. The HAND2, ISL1 and TBX2 super-enhancers, along with high expression levels of their encoded mRNAs, were maintained after ATRA-mediated differentiation. Concomitantly, new super-enhancers were established within the MEIS1, HIC1 and SOX4 loci, which coincided with increased expression levels of these genes. Thus, 
bioRxiv preprint doi: https://doi.org/10.1101/2020.07.23.218834; this version posted July 24, 2020. The copyright holder for this preprint (which

was not certified by peer review) is the author/funder, who has granted bioRxiv a license to display the preprint in perpetuity. It is made available under aCC-BY-NC-ND 4.0 International license.

ATRA initiates a change in cell state of neuroblastoma cells by replacing the adrenergic CRC with a retino-sympathetic CRC, which also includes the RARA, RARB, HAND2, ISL1, TBX2 and TBX3 transcription factors. The retino-sympathetic CRC then drives the expression of an extended regulatory network that enforces the differentiation of neuroblasts into mature sympathetic neuronal cells.

In a companion study by Banerjee and Gryder et al., the authors independently observed that the shift from self-renewal to differentiation in neuroblastoma involves loss of GATA3 expression. The DNA-binding activity of the GATA3 transcription factor is required to establish a super-enhancer that regulates the LMO1 gene locus in a subset of neuroblastoma tumors and cell lines, due to an inherited predisposition allele (rs2168101, G>T) that creates a GATA binding motif ${ }^{38}$. This leads to high levels of GATA3-dependent expression of LMO1, which then becomes a dependency gene in these cells and functions as a transcriptional co-regulator of the adrenergic $C R C^{14}$. Loss of GATA3 expression following treatment with ATRA resulted in collapse of the LMO1 super-enhancer in KCNR cells (MYCNamplified), highlighting the interconnected regulation of transcription factors and co-regulators within the adrenergic CRC. Banerjee and Gryder et al. also study the time course of the ATRA-induced switch from the adrenergic $\mathrm{CRC}$ to the new retino-sympathetic $\mathrm{CRC}$ in their paper, while we include gene specific ChIP-seq for key transcription factors RARA and MEIS1, establishing the interconnected regulation mediated by the new $\mathrm{CRC}$ that drives sympathetic neuronal differentiation. Interestingly, our analysis shows that the RARB gene is coordinately upregulated and acquires a new super-enhancer as a result of ATRA treatment, which is consistent with earlier work from Thiele and coworkers showing that coordinate activation of RARA and RARB is required to mediate ATRA-mediated neuroblastoma cell growth arrest ${ }^{39}$.

Retinoids are vitamin A derivatives that have an essential function in vertebrate development by regulating gene expression programs, including a major role in specification of the nervous system ${ }^{40,41}$. Both isotretinoin and ATRA are capable of binding to RAR receptors ${ }^{42,43}$, including RARA, which is driven by a super-enhancer and is highly expressed as a target of the adrenergic CRC and as a functional member of the retino-sympathetic CRC. Upon treatment, ATRA binds to RARA and RARB, 
and along with RXR the complexes bind to their response elements in enhancers and promoters to activate the expression of genes associated with neuroblastoma differentiation ${ }^{44}$. Our findings indicate that ATRA bound RARA acts as a potent activator and becomes an integral component of the retinosympathetic CRC. Thus, the differentiation of neuroblastoma cells treated with ATRA likely reflects transcriptional reprogramming that occurs in response to endogenous retinoids during the normal maturation of migratory neuroblasts into non-proliferative sympathetic neurons ${ }^{41}$.

Collapse of the adrenergic CRC mediated by ATRA includes marked downregulated expression of the amplified MYCN oncogene, which must be suppressed prior to neuroblast differentiation ${ }^{36}$. This is achieved in neuroblastomas with $M Y C N$ gene amplification because endogenous cis-regulatory elements included in the $M Y C N$ amplicon are selectively activated by the adrenergic $\mathrm{CRC}^{30}$. This likely explains the physiologic expression of $M Y C N$ observed in non-transformed adrenergic neuroblasts that serve as the cell of origin for this from of neuroblastoma, and is supported by the theory that genomic amplification events require active gene expression in order to be selected for in tumorigenesis; such as the amplification of basal levels of active $M Y C N$ expression in immature neuroblasts leading to neuroblastoma ${ }^{45}$.

MYCN gene amplification is the most recurrent form of $M Y C$ family gene activation in neuroblastoma - but it is not the only mechanism. Neuroblastoma tumors can also activate the expression of MYCN or MYC by chromosomal structural rearrangements that hijack super-enhancers regulating the expression of $H A N D 2^{11}$, which is an important transcription factor in both the adrenergic and retino-sympathetic CRCs. Because HAND2 is a member of both CRCs, the super-enhancer regulating it is active in both cell states. Its super-enhancer from the intact allele drives HAND2 expression while the super-enhancer on the translocated allele continues to drive expression of $M Y C N$ as the retino-sympathetic CRC is attempting to form during ATRA treatment. Therefore, MYCN expression in these cells is not ablated by treatment with ATRA, preventing MYCN downregulation, which is essential for cell cycle arrest and differentiation ${ }^{27}$. We have previously shown that MYC can replace $M Y C N$ in neuroblastoma transformation when $M Y C$ is hijacked and becomes regulated by the 
translocated HAND2 super-enhancer ${ }^{11}$. Thus, high levels of expression of either MYCN or MYC due to translocations with the HAND2 locus produce neuroblastoma cells that are resistant to the effects of ATRA in inducing neuroblastoma cell differentiation. Examples of this resistance phenotype include the cell lines NBL-S, with $t(2 ; 4)$ activating $M Y C N$, and SKNAS, with $t(4 ; 8)$ activating $M Y C$. In both cases, ATRA-treated cells retain high levels of $M Y C N$ or $M Y C$ oncogene expression at the RNA and protein levels and are blocked from undergoing differentiation. These findings highlight a resistance mechanism that could explain why some patients may not benefit from maintenance therapy with retinoids. Thus, our studies of transcriptional control of cell state in neuroblastoma not only provide insight into the role of ATRA in the treatment of high-risk neuroblastoma, but also reveal mechanisms that impart resistance to retinoids in some children with this disease.

\section{METHODS}

\section{Cell lines and proliferation assays}

BE2C and SKNAS cells were purchased from ATCC; NGP and NBL-S cells were purchased from DSMZ. All neuroblastoma cell lines were cultured at $5 \% \mathrm{CO}_{2}$ in RPMI media containing $10 \%$ FBS and 1\% Penicillin-Streptomycin. Cells were routinely tested (every 3 months) for mycoplasma contamination and genotyped (every 12 months) by STR analysis at the Dana-Farber Molecular Diagnostic Core Facility. Cell proliferation was measured by plating 5000 cells per well in white 96-well plates in 100 uL of total media containing DMSO or $5 \mu \mathrm{M}$ ATRA. Cell viability at each time point was assayed with Cell Titer glo (Promega) according to the manufacturer's protocol.

\section{Compounds and reagents}

Isotretinoin (13-cis retinoic acid) and ATRA (all-trans retinoic acid) were purchased from Selleckchem. Cell culture grade DMSO was purchased from ATCC. Compounds were resuspended in DMSO to a stock concentration of $100 \mathrm{mM}$ and added directly to cell culture media or zebrafish water at the indicated concentrations. 


\section{Zebrafish tumorigenesis assays}

Transgenic zebrafish were developed as previously reported ${ }^{9}$. All animal experiments were conducted at Dana-Farber Cancer Institute in accordance with animal care and use committee protocol \#02-107. Wildtype and transgenic zebrafish were maintained under standard aquaculture conditions at the DanaFarber Cancer Institute. Transgenic $d b h: M Y C N$ zebrafish were crossed to a stable $d b h: E G F P$ expressing line and sorted for EGFP+ fluorescence. Tumor-positive offspring were anesthetized with tricaine and subsequently monitored for neuroblastoma tumor progression. All comparative experimental groups for PSNS and neuroblastoma tissue quantification were imaged under the same conditions, and acquired fluorescent images were quantified using ImageJ software (NIH) by measuring the area of EGFP fluorescence. For neuroblastoma tumor-size quantification, the fluorescent area was normalized against the surface area of the fish head as fish size was variable. Overlays were created using ImageJ and Adobe Photoshop 7.0.1.

\section{Immunofluorescence and confocal microscopy}

Cell were grown and compound treated on glass slides in 6-well cell culture plates. After 6 days of treatment with DMSO or ATRA, slides were incubated with a primary antibody at $4^{\circ} \mathrm{C}$ overnight, washed with PBST, and then incubated with a secondary antibody for 2 hours at room temperature. Secondary antibodies were conjugated with Alexa Fluor 488 (Life Technologies). Alexa Fluor 568 Phalloidin (Life Technologies) and DAPI (BD Biosciences) were used for counter staining. Fluorescent images were taken with a Leica SP5X scanning confocal microscope at the Confocal and Light Microscopy core facility at Dana-Farber Cancer Institute.

\section{Western blotting}

Protein samples were collected and lysed using RIPA buffer containing protease and phosphatase inhibitors (Cell Signaling Technology). Lysates were quantified by Bradford assay (Bio-rad), and $10 \mu \mathrm{g}$ 
of extracted protein was separated using Novex SDS-PAGE reagents and transferred to nitrocellulose membranes (Life Technologies). Membranes were blocked in 5\% milk protein and incubated with primary antibodies overnight followed by secondary HRP-linked goat anti-rabbit and anti-mouse (Cell Signaling) antibodies (1:1000) according to the manufacturers' instructions. Antibody bound membranes were incubated with SuperSignal West Pico chemiluminescent substrate (Thermo-Fisher) and developed using HyBlot CL autoradiography film (Thomas Scientific). The antibodies used immunoblotting are listed in the following table:

\begin{tabular}{|l|l|l|l|l|}
\hline Antibody & Assay & Manufacturer & Catalog \# & Lot \# \\
\hline H3K27ac & ChIP-seq & Abcam & ab4729 & GR3211959-1 \\
\hline H3K27me3 & ChIP-seq & Abcam & ab192985 & GR3204355-5 \\
\hline RARA & ChIP-seq & Abcam & ab41934 & GR298513-2 \\
\hline MEIS1 & ChIP-seq & Abcam & ab19867 & GR153319-2 \\
\hline MYCN & Western blot & Cell Signaling & 9045 & 2 \\
\hline MYC & Western blot & Cell Signaling & 5605 & 8 \\
\hline Histone H3 & Western blot & Cell Signaling & 4499 & 9 \\
\hline HAND2 & Western blot & Santa Cruz & sc-398167 & K1716 \\
\hline ISL1 & Western blot & DSHB & 39.4 D5 & $1-4-18$ \\
\hline PHOX2B & Western blot & Santa Cruz & sc-376993 & E3012 \\
\hline GATA3 & Western blot & Cell Signaling & 5852 & 5 \\
\hline ALK & Western blot & Cell Signaling & 3333 & 11 \\
\hline TUBB3 & Western blot & Cell Signaling & 5568 & 7 \\
\hline ACTB & Western blot & Cell Signaling & 4070 & 18 \\
\hline FN1 & IF & Cell Signaling & 26836 & 1 \\
\hline TUBB3 & IF & Cell Signaling & 5568 & 7 \\
\hline VIM & IF & Cell Signaling & 5741 & 5 \\
\hline
\end{tabular}

\section{Spike-in normalized RNA-seq}

DMSO and ATRA treated cells were grown in triplicate using 6-well plates and collected directly into

Trizol. ERCC spike-in RNA was diluted 1:10 in nuclease-free water and added directly to Trizol lysates after being normalized to cell number as per the manufacturer's protocol (Life Technologies). Libraries were prepared using Illumina TruSeq stranded specific sample preparation kits from 500ng of purified total RNA according to the manufacturer's protocol. The finished dsDNA libraries were quantified by Qubit fluorometer (Thermo-Fisher), TapeStation 4200 (Agilent), and RT-qPCR using the Kapa Biosystems library quantification kit (Roche) according to manufacturer's protocols. Indexed libraries 
were pooled in equimolar ratios and sequenced on an Illumina NextSeq 550 with single-end $75 \mathrm{bp}$ reads by the Dana-Farber Cancer Institute Molecular Biology Core Facilities. Reads were aligned to a reference genome containing the non-random chromosomes from hg19 and the sequences of ERCC probes using hisat2 with parameters --no-novel-juncs and -G set to a gene database file downloaded from RefSeq on 7/5/2017 to which positions of the ERCC probes were added. Coverage of the genes in this list was calculated using htseq-count with parameters -i gene_id --stranded=reverse $-f$ bam -m intersection-strict. Violin plots were created using Prism 7.01 (GraphPad).

\section{Histone and transcription factor ChIP-seq}

Chromatin Immunoprecipitation coupled with high-throughput DNA sequencing (ChIP-seq) was performed as previously described. For each ChIP, $5 \mu \mathrm{g}$ of antibody coupled to $2 \mathrm{ug}$ of magnetic Dynabeads (Life Technologies) were added to $3 \mathrm{~mL}$ of sonicated nuclear extract from formaldehyde fixed cells. Chromatin was immunoprecipitated overnight, crosslinks were reversed, and DNA was purified by precipitation with phenol:chloroform:isoamyl alcohol. DNA pellets were resuspended in 25 $\mathrm{UL}$ of TE buffer. Illumina sequencing, library construction and ChIP-seq analysis methods were previously described. ChIP-seq datasets will be available in NCBI-GEO.

\section{Genome-wide occupancy analysis}

Reads were aligned to the human genome (hg19) using bowtie with parameters $-\mathrm{k} 2-\mathrm{m} 2$-e 70 -best and $-\mathrm{I}$ set to the read length. For visualization, WIG files were created from aligned ChIP-Seq read positions using MACS with parameters $-\mathrm{w}-\mathrm{S}-\mathrm{space}=50$-nomodel - shiftsize $=200$ to artificially extend reads to be $200 \mathrm{bp}$ and to calculate their density in $50 \mathrm{bp}$ bins. Read counts in $50 \mathrm{bp}$ bins were then normalized to the millions of mapped reads, giving reads per million (rpm) values. WIG files were visualized in the IGV browser version 2.7.2. Constituent enhancers were identified using MACS using input control and with parameters-p 1e-9 and -keep-dup 1 or $-\mathrm{p} 1 \mathrm{e}-9$ and -keep-dup all. The union of both peak calls was used as input for ROSE (https://bitbucket.org/young_computation/rose/). ROSE 
separated SEs from typical enhancers with parameters -s 12,500 -t 2,000. Heatmaps were constructed in the $4 \mathrm{~kb}$ regions centered on the collapsed union of RARA and MEIS1 binding sites separately defined in each cell line using MACS using input control and -p 1e-9. Read density was calculated in 50 bins per region using bamToGFF with parameters $-d-r-m 50$.

\section{Cell cycle analysis}

Cells were treated with DMSO or ATRA for 6 days in triplicate, and 500,000 cells per sample were collected and lysed in cold hypotonic propidium iodide (PI) / RNase solution ( $50 \mathrm{ug} / \mathrm{mL} \mathrm{PI}, 4 \mathrm{mM}$ sodium citrate, $30 \mathrm{U} / \mathrm{mL}$ RNaseA, 0.1\% TX-100). Samples were then vigorously vortexed and incubated at $37^{\circ} \mathrm{C}$ for $10 \mathrm{~min}$. Sodium chloride was added to a final concentration of $0.15 \mathrm{M}$, and stained nuclei were stored at $4^{\circ} \mathrm{C}$ until analysis was ready to be performed. The samples were then analyzed by flow cytometry (BD LSRFortessa). Cell cycle distribution was analyzed with the FlowJo cell cycle Watson (Pragmatic) model. The singlet population was isolated with a live cell gate. To analyze the proportion of cells in G1, S, and G2/M, the Watson (Pragmatic) model with the $\mathrm{G} 2$ peak constrained on $\mathrm{G} 1=\mathrm{G} 2 \mathrm{x}$ 2 was used. Both debris and doublets were removed from the analysis.

\section{Statistical analysis}

Statistical calculations were performed using Prism 7.01 (GraphPad). Digital images of the fluorescence signal for transgenic embryos, and the area of the fluorescence coverage, was quantified with ImageJ $(\mathrm{NIH})$ for Fig. 1. Multivariate ANOVA analysis followed by two-tailed, unpaired t-tests with confidence intervals of $95 \%$ were used for the quantitative assays.

\section{ACKNOWLEDGEMENTS}

We would like to thank J.R. Gilbert for editorial assistance and critical comments and Z. Herbert of the Dana-Farber Molecular Biology Core Facility for genomics support. This work was supported by NIH 
grants R35CA210064 (A.T.L.) and R01CA216391 (J.Z.). M.W.Z. was supported by grants from the Alex's Lemonade Stand Foundation, Charles A. King Trust, and Claudia Adams Barr Program. A.D.D. was supported by grants from the Alex's Lemonade Stand Foundation, CureSearch for Children's Cancer and Damon Runyon-Sohn Foundation. F.O. acknowledges the German Cancer Aid for their generous funding within the Mildred-Scheel-Postdoctoral program of the Mildred Scheel Foundation. B.J.A. is supported by the American Lebanese Syrian Associated Charities (ALSAC).

\section{AUTHOR CONTRIBUTIONS}

M.W.Z., A.D.D., S.H., R.A.Y., B.J.A. and A.T.L. conceived the project, performed data interpretation and wrote the manuscript with input from all authors. M.W.Z. and A.D.D. designed and performed ChIPseq, RNA-seq and flow cytometry experiments. B.J.A. performed ChIP-seq and RNA-seq computational analysis. Y.L. and J.Z. analyzed neuroblastoma cell line genomic data. M.W.Z. and S.H. designed and performed in vivo zebrafish experiments. F.O., H.S. and T.T. performed cellular immunofluorescence assays and confocal microscopy. A.B. and Y.L. performed western blotting and other experiments.

\section{COMPETING INTERESTS}

B.J.A. is a shareholder in Syros Pharmaceuticals. R.A.Y. is a shareholder in Syros Pharmaceuticals and is a consultant/advisory board member for the same. The other authors declare no competing interests. 


\section{REFERENCES}

1. Hnisz, D. et al. Super-enhancers in the control of cell identity and disease. Cell 155, 934-947 (2013).

2. Saint-André, V. et al. Models of human core transcriptional regulatory circuitries. Genome Res. 26, 385-396 (2016).

3. Arendt, D. et al. The origin and evolution of cell types. Nat. Rev. Genet. 17, 744-757 (2016).

4. Kanki, Y. et al. Dynamically and epigenetically coordinated GATA/ETS/SOX transcription factor expression is indispensable for endothelial cell differentiation. Nucleic Acids Res. 45, 4344-4358 (2017).

5. Huang, M. et al. dbCoRC: a database of core transcriptional regulatory circuitries modeled by H3K27ac ChIP-seq signals. Nucleic Acids Res. 46, D71-D77 (2018).

6. Martik, M. L. \& Bronner, M. E. Regulatory Logic Underlying Diversification of the Neural Crest. Trends Genet. TIG 33, 715-727 (2017).

7. Matthay, K. K. et al. Neuroblastoma. Nat. Rev. Dis. Primer 2, 16078 (2016).

8. Huber, K. The sympathoadrenal cell lineage: specification, diversification, and new perspectives. Dev. Biol. 298, 335-343 (2006).

9. Zhu, S. et al. Activated ALK collaborates with MYCN in neuroblastoma pathogenesis. Cancer Cell 21, 362-373 (2012).

10. Morrison, M. A., Zimmerman, M. W., Look, A. T. \& Stewart, R. A. Studying the peripheral sympathetic nervous system and neuroblastoma in zebrafish. Methods Cell Biol. 134, 97-138 (2016).

11. Zimmerman, M. W. et al. MYC Drives a Subset of High-Risk Pediatric Neuroblastomas and Is Activated through Mechanisms Including Enhancer Hijacking and Focal Enhancer Amplification. Cancer Discov. 8, 320-335 (2018).

12. Boeva, V. et al. Heterogeneity of neuroblastoma cell identity defined by transcriptional circuitries. Nat. Genet. 49, 1408-1413 (2017). 
13. van Groningen, T. et al. Neuroblastoma is composed of two super-enhancer-associated differentiation states. Nat. Genet. 49, 1261-1266 (2017).

14. Wang, L. et al. ASCL1 is a MYCN- and LMO1-dependent member of the adrenergic neuroblastoma core regulatory circuitry. Nat. Commun. 10, 5622 (2019).

15. Soldatov, R. et al. Spatiotemporal structure of cell fate decisions in murine neural crest. Science 364, (2019).

16. Zeid, R. et al. Enhancer invasion shapes MYCN-dependent transcriptional amplification in neuroblastoma. Nat. Genet. 50, 515-523 (2018).

17. Chipumuro, E. et al. CDK7 inhibition suppresses super-enhancer-linked oncogenic transcription in MYCN-driven cancer. Cell 159, 1126-1139 (2014).

18. Durbin, A. D. et al. Selective gene dependencies in MYCN-amplified neuroblastoma include the core transcriptional regulatory circuitry. Nat. Genet. 50, 1240-1246 (2018).

19. Villablanca, J. G. et al. Phase I trial of 13-cis-retinoic acid in children with neuroblastoma following bone marrow transplantation. J. Clin. Oncol. Off. J. Am. Soc. Clin. Oncol. 13, 894-901 (1995).

20. Reynolds, C. P. et al. Comparison of 13-cis-retinoic acid to trans-retinoic acid using human neuroblastoma cell lines. Prog. Clin. Biol. Res. 385, 237-244 (1994).

21. Matthay, K. K. et al. Treatment of high-risk neuroblastoma with intensive chemotherapy, radiotherapy, autologous bone marrow transplantation, and 13-cis-retinoic acid. Children's Cancer Group. N. Engl. J. Med. 341, 1165-1173 (1999).

22. Matthay, K. K. et al. Long-term results for children with high-risk neuroblastoma treated on a randomized trial of myeloablative therapy followed by 13-cis-retinoic acid: a children's oncology group study. J. Clin. Oncol. Off. J. Am. Soc. Clin. Oncol. 27, 1007-1013 (2009).

23. Chen, M.-C., Hsu, S.-L., Lin, H. \& Yang, T.-Y. Retinoic acid and cancer treatment. BioMedicine 4, 22 (2014). 
24. Sidell, N., Altman, A., Haussler, M. R. \& Seeger, R. C. Effects of retinoic acid (RA) on the growth and phenotypic expression of several human neuroblastoma cell lines. Exp. Cell Res. 148, 21-30 (1983).

25. Reynolds, C. P., Matthay, K. K., Villablanca, J. G. \& Maurer, B. J. Retinoid therapy of high-risk neuroblastoma. Cancer Lett. 197, 185-192 (2003).

26. Thiele, C. J., Deutsch, L. A. \& Israel, M. A. The expression of multiple proto-oncogenes is differentially regulated during retinoic acid induced maturation of human neuroblastoma cell lines. Oncogene 3, 281-288 (1988).

27. Thiele, C. J., Reynolds, C. P. \& Israel, M. A. Decreased expression of N-myc precedes retinoic acid-induced morphological differentiation of human neuroblastoma. Nature 313, 404-406 (1985).

28. Whyte, W. A. et al. Master transcription factors and mediator establish super-enhancers at key cell identity genes. Cell 153, 307-319 (2013).

29. Kouzarides, T. Chromatin modifications and their function. Cell 128, 693-705 (2007).

30. Morton, A. R. et al. Functional Enhancers Shape Extrachromosomal Oncogene Amplifications. Cell 179, 1330-1341.e13 (2019).

31. Westermark, U. K., Wilhelm, M., Frenzel, A. \& Henriksson, M. A. The MYCN oncogene and differentiation in neuroblastoma. Semin. Cancer Biol. 21, 256-266 (2011).

32. Chlapek, P., Slavikova, V., Mazanek, P., Sterba, J. \& Veselska, R. Why Differentiation Therapy Sometimes Fails: Molecular Mechanisms of Resistance to Retinoids. Int. J. Mol. Sci. 19, (2018).

33. Matsumoto, K., Lucarelli, E., Minniti, C., Gaetano, C. \& Thiele, C. J. Signals transduced via insulinlike growth factor I receptor (IGF(R)) mediate resistance to retinoic acid-induced cell growth arrest in a human neuroblastoma cell line. Cell Death Differ. 1, 49-58 (1994).

34. Wei, S.-J. et al. MYC transcription activation mediated by OCT4 as a mechanism of resistance to 13-cisRA-mediated differentiation in neuroblastoma. Cell Death Dis. 11, 368 (2020).

35. Sidell, N. Retinoic acid-induced growth inhibition and morphologic differentiation of human neuroblastoma cells in vitro. J. Natl. Cancer Inst. 68, 589-596 (1982). 
36. Wada, R. K. et al. Cell type-specific expression and negative regulation by retinoic acid of the human N-myc promoter in neuroblastoma cells. Oncogene 7, 711-717 (1992).

37. Reynolds, C. P. et al. Response of neuroblastoma to retinoic acid in vitro and in vivo. Prog. Clin. Biol. Res. 366, 203-211 (1991).

38. Oldridge, D. A. et al. Genetic predisposition to neuroblastoma mediated by a LMO1 super-enhancer polymorphism. Nature 528, 418-421 (2015).

39. Giannini, G., Dawson, M. I., Zhang, X. \& Thiele, C. J. Activation of three distinct RXR/RAR heterodimers induces growth arrest and differentiation of neuroblastoma cells. J. Biol. Chem. 272 , 26693-26701 (1997).

40. Blumberg, B. et al. An essential role for retinoid signaling in anteroposterior neural patterning. Dev. Camb. Engl. 124, 373-379 (1997).

41. Janesick, A., Wu, S. C. \& Blumberg, B. Retinoic acid signaling and neuronal differentiation. Cell. Mol. Life Sci. CMLS 72, 1559-1576 (2015).

42. Giguere, V., Ong, E. S., Segui, P. \& Evans, R. M. Identification of a receptor for the morphogen retinoic acid. Nature 330, 624-629 (1987).

43. Petkovich, M., Brand, N. J., Krust, A. \& Chambon, P. A human retinoic acid receptor which belongs to the family of nuclear receptors. Nature 330, 444-450 (1987).

44. Carpentier, A. et al. Distinct sensitivity of neuroblastoma cells for retinoid receptor agonists: evidence for functional receptor heterodimers. Oncogene 15, 1805-1813 (1997).

45. Furlan, A. et al. Multipotent peripheral glial cells generate neuroendocrine cells of the adrenal medulla. Science 357, (2017). 